\title{
Vinculação aos pais e vinculação amorosa: esperança e satisfação com a vida
}

\section{Attachment to parents and romantic attachment: relations with hope and life satisfaction}

\author{
Teresa Sousa Machado, Cátia Dias-da-Costa, José Tomás da Silva \\ *FPCE-UC
}

\begin{abstract}
Resumo
Representações das experiências precoces de vinculação estruturam novas relações significativas ao longo da vida. Estudam-se relações entre vinculação parental, vinculação amorosa, satisfação com a vida e orientação para o futuro, numa amostra de 262 jovens adultos (idades entre 18 e 34 anos; $\mathrm{M}=21.3 ; \mathrm{DP}=3.12$ ). Recorreu-se ao Inventário de Vinculação aos Pais e Pares (IPPA), Questionário de Vinculação Amorosa (QVA), Escala Satisfação com a Vida (SWL), e Escala de Esperança (EE). Os dois tipos de vinculação relacionam-se moderadamente, e a satisfação com a vida e orientação para o futuro, são explicados pela vinculação parental (mas não pela amorosa).

Palavras chave: vinculação parental, vinculação romântica, orientação para futuro, satisfação com vida.
\end{abstract}

\begin{abstract}
Representations about attachment previous experiences structure the quality of new relationships later in life. This paper analyses the relations between attachment to parents, romantic attachment, life satisfaction and future orientation (hope), in 262 young adults (aged 18-to-34 years old; $\mathrm{M}=21.3$; DP=3.12). The Inventory of Parent and Peer Attachment (IPPA), the Romantic Attachment (QVP), Satisfaction with Life (SWL); and Hope Scale were used. The two kinds of attachment seem to be independent; SWL and future orientation variation are explained by attachment to parents (but not by romantic attachment).

Keywords: attachment to parents, romantic attachment, future orientation, life satisfaction
\end{abstract}

A construção de vinculações a cuidadores e parceiros mantém-se, ao longo da história do Homem, pelo valor adaptativo para a sobrevivência e proteção na vulnerabilidade. Saber se os padrões de adaptação emergem de forma coerente, passo-a-passo, desde a infância, é uma questão fundamental na psicologia do desenvolvimento (Simpson, \& Rholes, 2012; Sroufe, 2005); tendo sido tratada por Bowlby (1982; 1988), ao propor que as diferenças nas representações das relações significativas emergem na história das interações, ou seja, não derivam de fantasias. A qualidade das vinculações é fundamental para o desenvolvimento da personalidade, e a teoria assenta na importância das experiências de vida, contrariando a interpretação psicanalítica clássica, embora admita que a interiorização dessas experiências está imbuída de interpretações pessoais, que dão origem aos modelos operantes internos. Os modelos estruturam-se a partir do momento em que a criança tem a noção de "permanência do objecto", ou seja, quando pode recorrer, mentalmente, à figura do outro, mesmo quando ausente (Piaget, 1947; 1948; Waters, \& Waters, 2006). A "continuidade" nos estilos de relações traduz a existência desses modelos mentais do self, dos outros, e das relações, sendo que tanto as "formas saudáveis de amor como as não saudáveis têm origem em adaptações a condições sociais específicas" (Hazen, \& Shaver, 1987, p.511), pelo que são analisáveis, interpretáveis e passíveis de reinterpretações.

Os modelos internos funcionam como uma espécie de scrpits (ou esquemas) que geram expectativas que preparam para acção (Colin, 1996). São como "um conjunto de regras conscientes e/ou inconscientes que organizam a informação relevante para a vinculação" (Delius, Bovenschen, \& Spangler, 2008, p. 396). Construídos numa fase precoce do desenvolvimento, em que o pensamento não é "operatório", os modelos internos facilmente se tornam automáticos, justificando que se perpetuem disfunções anteriores, ou, noutro sentido, a anterior adaptabilidade (Sroufe, 2005). Alterações nas relações e o acesso ao raciocínio formal (hipotético-dedutivo e probabilístico) possibilitam novas interpretações, permitindo actualizações das representações da vinculação (Hamilton, 2000; Kesselring, 1992; Machado 2003; Piaget, 1948).

Contrariamente às ideias freudianas sobre a continuidade desenvolvimental criança-adulto (pelas fixações e regressões), a teoria da vinculação propõe que o desenvolvimento social envolve sempre uma construção contínua, revisões, integrações e abstrações progressivas dos modelos mentais. Esta análise é consentânea com interpretações piagetianas que mostram como formas mais complexas do raciocínio possibilitam reinterpretações do mundo e das relações (passadas e presentes); justificando-se que Bowlby tenha recorrido a este modelo para explicitar implicações da vinculação ao longo da vida (Pallini, \& Barcaccia, 2014).

As possibilidades de revisão não são porém iguais para todos os sujeitos, sabendo-se que em amostras de adolescentes e jovens adultos de baixo-a-moderado risco, a segurança tende a ser relativamente estável, mas é-o bastante menos em amostras de risco (Beijersbergen, Juffer, Bakermans-Kranenburg, \& van Ijzendoorn, 2012; Scott, Whalen, Zalewski, Beeney, Pilkonis, Hipwell, \& Stepp, 2013). Reforçando a tese de Simpson e Rholes (2012), que conceptualiza a insegurança na vinculação 
como uma disposição que tende a gerar adaptações interrelacionais disfuncionais, em momentos stressantes da vida.

Desde os anos 1970, vários investigadores recorrem à teoria de Bowlby para analisar a natureza e etiologia da das relações amorosas, destacando-se os trabalhos de Shaver e Hazan (Fraley, \& Shaver, 2000). Estes observaram como as dinâmicas das relações amorosas são similares às das relações das crianças com os cuidadores nas implicações da segurança, certeza na acessibilidade e responsividade do parceiro, e recurso ao mesmo enquanto fonte de segurança (Bretherton, \& Munholland, 2008; Fraley, \& Shaver, 2000). Sugerem ainda que os três padrões descritos por Ainsworth (seguro, ansioso-ambivalente e evitamento) também se aplicam às relações românticas. Porém, o maior consenso é de que a continuidade entre vinculação aos pais e vinculação romântica é reduzida (Pietromonaco \& Barrett, 2000). O que não invalida que as emoções e comportamentos que caracterizam ambas as relações (românticas e pais-criança) sejam similares em termos do que motiva a activação e terminus do comportamento de vinculação (Fraley \& Shaver, 2000). Isto é, não necessitam obrigatoriamente da proximidade em momentos em que tudo "está bem", e procuram a proximidade perante a angústia.

$\mathrm{Na}$ infância o comportamento de vinculação, i.e., a procura activa do outro, é adaptativo, estando o cuidador geralmente disponível para proteger; no amor romântico, o comportamento de vinculação é apenas uma das componentes, a par de outros sistemas comportamentais (como o sexual e o reprodutivo), distintos em termos teóricos (Fraley \& Shaver, 2000). As transições para as relações adultas, são caracterizadas por maior equilíbrio (do que na infância) entre autonomia e proximidade, correlacionando-se esse equilíbrio com a satisfação com a vida (Chopik, Edelstein, van Anders, Wardecker, Shipman, \& Samples-Steele, 2014; Guarnieri, Smorti, \& Tani, 2015).

A questão da influência dos modelos primários na construção de novas relações mantém a actualidade empírica e teórica - não obstante as alterações nas tipologias familiares ao longo dos tempos. Quanto às relações entre vinculação parental e vinculação romântica, alguns estudos mostram relações significativas entre ambas, e.g., adolescentes e/ou adultos mais seguros relativamente às figuras parentais relacionam-se de modo mais seguro com o par romântico (Gleeson \& Fitzgerald, 2014; Matos \& Costa, 2006; Muraru \& Turluc, 2012), mantêm relações mais duradoiras e recorrem a mais adjectivos positivos para descrever os parceiros (Vorria, Vairami, Gialaouzidis, Kotroni, Markou, Marti, et al., 2007).

Outros reportam uma fraca correlação entre um ajustamento marital pobre e dificuldades anteriores nas relações com os pais; e ainda que a percepção de segurança aos pais não prediz a qualidade das relações românticas posteriores (Dinero, Conger, Shaver, Widaman, \& Larsen-Rife, 2008; Twedt, 2013), dados aparentemente inconsistentes com as expectativas teóricas sobre a segurança na vinculação. Apostolidou (2006) não encontra relações significativas entre o estilo de vinculação aos pais e o estilo nas relações românticas em jovens adultos; embora verifique uma correlação positiva significativa entre percepção de controlo pela mãe e a ansiedade na relação romântica (nos homens). $\mathrm{O}$ que sugere que serão aspectos particulares das relações (eventualmente com raízes na história do sujeito) que influenciam a dinâmica (mais imediata) de novas relações. A natureza dos modelos internos justifica as diferenças das múltiplas influências: tratam-se de estruturas cognitivas que reflectem percepções cumulativas ao longo do desenvolvimento; incorporando memórias semânticas, episódicas, afectivas, regras de conduta e de pensamento, crenças, atitudes, valores e expectativas sobre parceiros, entre outras (Mikulincer \& Shaver, 2007; Simpson, \& Rholes, 2012).

A ausência de acordo nos resultados publicados aponta a necessidade de mais estudos, nomeadamente para que se teste a hipótese de Mikulincer e Shaver (2007), de que relações posteriores poderão influenciar mudanças no estilo de vinculação do sujeito, impulsionando um afastamento do padrão inicial; e que não só a relação de vinculação aos pais interfere na interiorização dos modelos internos, mas também as interpretações (e fantasias) que a criança faz do que observou da relação entre os pais contribuirá para a interiorização do que é expectável nas relações românticas (Bandura, 1977; Hare, Miga, \& Allen, 2009; Matos, \& Costa, 2006). Com efeito, Benson et al., (1993) mostram a influência de variáveis das dinâmicas da família de origem (como a triangulação, fusão, controlo) na qualidade das relações românticas mais tarde.

Cerca de 25 anos de pesquisa têm identificado correlações dos padrões de vinculação na infância com comportamentos posteriores, sendo que os sujeitos seguros apresentam maior tendência para "desactivarem" a necessidade de vinculação (pois não precisam de a testar constantemente); estão mais livres para investirem noutras tarefas, funcionarem melhor e terem relações românticas mais estáveis (Simpson \& Rholes, 2012). Importa analisar como as representações da vinculação aos pais se relacionam com as representações da vinculação romântica (em jovens adultos portugueses). E averiguar se não é a interiorização mais global da vinculação aos pais que sustém, com maior peso, a (in)adaptação no desenvolvimento, expressa por exemplo, na satisfação com a vida e orientação para o futuro. Tentando responder a estas questões, apresentamos um estudo exploratório das relações entre representações da vinculação aos pais e vinculação romântica, com a satisfação com a vida e orientação para o futuro, em jovens adultos.

\section{Método}

\section{Participantes}

A amostra é constituída por 262 jovens adultos, estudantes de instituições do Ensino Superior, de Coimbra, com idades entre os 18 e 34 anos $(M=21.3$; $\mathrm{DP}=3.12)$; sendo $\mathrm{N}=131(50 \%)$ raparigas e $50 \%$ rapazes; 159 dos jovens $(60.7 \%)$ encontravam-se no $3^{\circ}$ ou $4^{\circ}$ ano de um curso superior, e 103 (39.3\%) no $1^{\circ}$ ou $2^{\circ}$ ano; 184 (70.1\%) frequentam cursos nas engenharias, e 78 
(29.9\%) cursos nas ciências sociais e humanas (psicologia, ciências da educação, serviço social, e direito). A maioria pertence ao nível socioeconómico médio $(\mathrm{N}=109 ; 41.8 \%)$, repartindo-se os restantes pelos níveis baixo ( $\mathrm{N}=99 ; 37.9 \% 9)$, e elevado $(\mathrm{N}=53 ; 20.2 \%)$.

\section{Instrumentos}

O IPPA - Inventory of Parent and Peer Attachment (Armsden \& Greenberg, 1987; versão portuguesa, de 28 itens) avalia as representações da vinculação global aos pais (e suas dimensões: confiança, comunicação, alienação), numa escala Likert de 5 pontos. As dimensões comunicação e confiança remetem para aspectos positivos de proximidade com os cuidadores, a dimensão alienação remete para representações de incompreensão e/ou isolamento relativamente aos pais. Um resultado global obtém-se pela soma da cotação comunicação e confiança, subtraindo a alienação.

O Questionário de Vinculação Amorosa (QVA) (Barbosa \& Costa, 2001), versão breve (25 itens), avalia a relação amorosa de adolescentes e jovens adultos, numa escala Likert de seis alternativas. São quatro as dimensões avaliadas da representação da relação amorosa ou do(a) parceiro(a) na relação: confiança, ambivalência, dependência e evitamento.

A Escala de Satisfação com a Vida (SWL), versão portuguesa de Simões (1992), avalia, em cinco itens, numa escala Likert de cinco níveis, o nível de satisfação global com a vida.

A Escala de Esperança - ou escala de futuro (EF) (Snyder, et al., 1991; 2003) - com doze itens (quatro dos quais são itens distratores) - avalia a orientação para o futuro, numa escala de oito alternativas. Para além do valor global "esperança global", distinguem-se duas dimensões (iniciativa e caminhos).

\section{Procedimento}

Os participantes foram recrutados em diferentes Faculdades, da Universidade de Coimbra, depois de obtida autorização dos docentes, tendo a aplicação ocorrido em sala de aula. A amostra foi controlada no sentido de só participarem sujeitos que têm experiência (presente ou passada) de relacionamento amoroso, tendo sido excluídos os (27 sujeitos) que reportam terem tido apenas "relacionamentos ocasionais".

\section{Resultados}

No que se refere às relações entre a representação da vinculação aos pais (IPPA) e a vinculação amorosa (QVA), os valores das correlações mostram-se significativos (com valores baixos a moderados).

A direção da relação apresenta-se no sentido esperado: positiva entre a dimensão confiança e o score global do IPPA e confiança do QVA; e negativas entre as dimensões confiança e comunicação do IPPA e dependência, evitamento e ambivalência do QVA (Tabela 1).

Tabela 1. Correlações de Pearson entre IPPA e QVA

\begin{tabular}{|c|c|c|c|c|}
\hline & \multicolumn{4}{|c|}{ QVA } \\
\hline & Confianç & Depend & Evitam. & Ambival. \\
\hline IPPA-Tot & $.17^{*}$ & $-.13 * *$ & - & $-.22^{*}$ \\
\hline
\end{tabular}

\begin{tabular}{lcccc} 
Confiança & $.12 * *$ & $-.17 *$ & - & $-.19^{*}$ \\
Comunica. & $.15 * *$ & - & - & $-.15^{* *}$ \\
Alienação & $-.21 *$ & - & - & $.25^{*}$ \\
\hline$* \mathrm{p}=0.01 ; \quad{ }^{* * \mathrm{p}}=0.05$ & &
\end{tabular}

Embora as correlações sejam significativas, e a direção ocorra no sentido esperado, a percentagem da variância explicada na relação destas variáveis é baixa: $4.8 \%$ da variância partilhada entre total IPPA e ambivalência (QVA), e 2.8\% da variância partilhada entre total IPPA e confiança (QVA). Entre a alienação (IPPA) e confiança (QVA) encontramos $4.4 \%$ da variância explicada, e entre alienação (IPPA) e ambivalência (QVA), encontramos $6.3 \%$ da variância partilhada.

Quanto às relações entre vinculação aos pais (IPPA) e satisfação com a vida (SWL), encontramos correlações positivas e estatisticamente significativas $(p \leq 0.01)$, entre as dimensões comunicação e confiança (.44 e .38, respectivamente), e negativa entre alienação e satisfação com a vida (-.45). Também entre a vinculação aos pais e a orientação para o futuro (escala esperança) as correlações são no sentido esperado: positivas entre IPPA total, confiança e comunicação e esperança, iniciativa e caminhos (EF), e negativas entre alienação (IPPA) e dimensões da EF; ou seja, no sentido esperado de acordo com a teoria da vinculação (Tabela 2.).

Tabela 2. Correlações entre IPPA, SWL, e EF

\begin{tabular}{ccccc}
\hline & & \multicolumn{3}{c}{ EF } \\
\cline { 2 - 5 } IPPA & (SWLS) & Esp & Ini & Caminhos \\
\cline { 2 - 5 } Confiança & .44 & .32 & .36 & .21 \\
Comunica & .38 & .25 & .28 & .17 \\
Alienação & -.45 & -.30 & -.35 & -.19 \\
Vinc[total] & .48 & .33 & .39 & .22 \\
\hline $\mathrm{p} \leq 0.01$ (bidirecional) & \multicolumn{3}{c}{}
\end{tabular}

Relativamente às relações entre vinculação amorosa (QVA) e satisfação com a vida (SWL), encontramos relações positivas baixas $(0.18 ; p \leq 0.01)$ entre a dimensão confiança e SWL, (com reduzida associação e variância explicada 3.2\%); e correlação negativa entre a dimensão ambivalência e SWL (-.27; 7.3\% variância explicada); as correlações entre as dimensões dependência e evitamento (QVA) com a SWL não alcançaram significância estatística. Em conformidade com o quadro teórico, a duração da relação amorosa potencia diferenças significativas nas dimensões do QVA (menor duração apresenta menor confiança ao par amoroso, sendo a magnitude do efeito moderada); a dependência é maior, sendo o efeito da diferença significativo, nas relações de duração entre 2 a 4 anos, comparativamente com as que variam entre 6 meses a 2 anos; e os que namoram há menos tempo são mais evitantes. Estar ou não envolvido numa relação romântica não mostrou ser revelante para a percepção da satisfação com a vida nestes jovens; assim como não o mostrou o tempo de duração da relação, ao contrário do que sugerem alguns autores (Diener, 1984; Guarnieri, et al., 2015).

\section{Discussão}

Globalmente, os dados reforçam a ideia de que representações de vinculações mais seguras aos pais se 
relacionam com maior nível de confiança na vinculação romântica (QVA); e que maior percepção de alienação aos pais se relaciona com maior ambivalência reportada na relação romântica, confirmando expectativas teóricas gerais. No entanto, os valores das correlações e da percentagem da variância da vinculação romântica, explicada sugerem, como alguns dos estudos revistos, que, também, na presente amostra os conceitos (vinculação parental vs. vinculação romântica) são relativamente independentes (Fraley \& Shaver, 2000; Mikulincer \& Shaver, 2007; Simpson \& Rholes, 2012). Em conformidade com o quadro teórico, a duração da relação amorosa potencia diferenças significativas nas dimensões do QVA, no sentido de aumento de confiança, diminuição de evitamento e de ambivalência, reforçando a ideia de que a formação de novos vínculos é um processo desenvolvimental.

As relações entre a vinculação aos pais e a satisfação com a vida (SWL) e orientação para o futuro (EF), apresentam-se mais robustas do que as relações entre a vinculação romântica e essas mesmas duas variáveis; mas as interpretações possíveis devem ter em conta a própria idade (fase desenvolvimento) dos sujeitos; em diferentes fases da vida, o peso atribuído a cada tipo de relação pode mudar - tendo tal um cariz adaptativo. $\mathrm{O}$ facto da vinculação aos pais, nestas idades, permitir a exploração - i.e., orientação para o futuro - confirma que estes mantêm, nesta fase, um peso importante para a segurança (a possibilidade de exploração pressupõe, segundo a teoria, a segurança interna) (Allen, 2013).

Neste trabalho analisaram-se efeitos e relações das representações da vinculação aos pais e vinculação ao par romântico em jovens adultos universitários; seria interessante confrontar estes dados com outros que analisassem as mesmas relações em amostras de risco i.e., com sujeitos reportando vinculações nitidamente inseguras ansiosas/ambivalentes com os pais - para testar a hipótese da maior variabilidade ao longo do desenvolvimento, nos padrões inseguros; e da eventual primazia de figuras diferentes de vinculação ao longo do desenvolvimento (Allen, 2013). O recurso apenas a instrumentos de auto-relato no presente estudo é um limite que implica que os resultados só podem ser considerados em termos de tendência (de relações) muito geral (não permitindo reacções espontâneas às questões de vinculação que, eventualmente, despoletassem inquietações nos sujeitos).

\section{Referencias}

Allen, J. G. (2013). Mentalizing in the development and treatment of attachment trauma. London: Karnac Books.

Apostolidou, Z. (2006). Are childhood experiences with parents linked to feelings in romantic relatioships during adulthood? The New School Psychology Bulletin, 4(1), 63-85.

Bandura, A. (1977). A social learning theory. Englewood Cliffs: Prentice-Hall.

Beijersbergen, M. D., Juffer, F., Bakermans-Kranenburg, M. J., \& van Ijzendoorn, 2012). Remaining or becoming secure: parental sensitive support predicts attachment quality from infancy to adolescence in a longitudinal adoption study. Developmental Psychology, 48(5), 1277-1282. doi: 10.1177/0963721412453588

Benson, M.J., Larson, J., Wilson, S. M., \& Demo, D. H. (1993). Family of origin influences on late adolescent romantic relationships. Journal of Marriage and the Family, 55, 663-672.

Bowlby, J. (1982). Attachment and loss- vol.I, Attachment. 2th ed. London: The Hogarth Press.

Bowlby, J. (1988). A secure base. Clinical applications of attachment theory. London: Routhedge.

Bretherton, I., \& Munholland, K. A. (2008). Internal working models in attachment relationships: Elaborating a central construct in attachment theory. In J. Cassidy, \& P. R. Shaver (Eds.), Handbook of attachment: Theory, research, and clinical applications ( $2^{\text {nd }}$ ed.), pp.102-127, New York: Guilford Press.

Chopik, W. J., Edelstein, R. S., van Anders, S. M., Wardecker B. M., Shipman, E. L., Samples-Steele, C. R. (2014). Too close for confort? Adult attachment and cuddling in romantic and parent-child relationships. Personality and Individual Differences, 69, 212-214. doi: 10.1016/j.paid.2014.05.35

Colin, V. L. (1996). Human attachment. Philadelphia: Temple University Press.

Delius, A., Bovenschen, I., \& Gottfried, S. (2008). The inner working model as a "theory of attachment": development during the preschool years. Attachment \& Human Development, 10(4), 395-414. doi: 10.1080/14616730802461425

Diener, E. (1984). Subjective well-being. Psychological Bulletin, 95(3), 542-575.

Dinero, R. E., Conger, R. D., Shaver, P. R., Widaman, K. F., \& Larsen-Rife, D. (2008). Influence of family of origin and adult romantic partners on romantic attachment security. Journal of Family Psychology, 22(4), 622-632. doi: 10.1037/a0012506

Fraley, R. C., \& Shaver, P. R. (2000). Adult romantic attachment: Theoretical developments, emerging controversies, and unanswered questions. Review of General Psychology, 4(2), 132-154. doi: 10.1037//1089-2680.4.2.132

Gleeson, G., \& Fitzgerald, A. (2014). Exploring the association between adult attachment styles in romantic relationships, perceptions of parents from childhood and relationship satisfaction. Health, 6, 1643-1661. doi: 10.4236/health.2014.613196

Guarnieri, S., Smorti, M., \& Tani, F. (2015). Attachment relationships and life satisfaction during emerging adulthood. Social Indicators Research, 121(3), 833-847. doi: 10.1007/s11205-014-0655-1

Hamilton, C. E. (2000). Continuity and discontinuity of attachment from infancy through adolescence. Child Development, 71, 690-694.

Hare, A. L., Miga, E. M., \& Allen, J. P. (2009). Intergenerational transmission of aggression in romantic relationships: the moderating role of attachment security. Journal of Family Psychology, 23(6), 808-818. doi: $10.1037 / \mathrm{a} 0016740$ 
Hazan, C., \& Shaver, P. (1987). Romantic love conceptualized as an attachment process. Journal of Personality and Social Psychology, 52 (3), 511-524.

Kesselring, T. (1992). Égocentrisme et équilibration. Y a-t-il une logique intrinsèque dans le développement mental? In D. Maurice, \& J. Montangero (Eds.), Équilibre et équilibration dans l'œuvre de Jean Piaget et au regard de courants actuels. Cahier $N^{\circ} 12$, Fondation Archives Jean Piaget.

Machado, T. S. (2003). Raciocínio operatório formal: O que se mantém da original definição piagetiana? Psychologica, 32, 147-169.

Machado, T. S. (2007). Padrões de vinculação aos pais em adolescentes e jovens adultos e adaptação à Universidade. Revista Portuguesa de Pedagogia, 41(2), 5-28.

Matos, P. M., \& Costa, M. E. (2006). Vinculação aos pais e ao par romântico em adolescentes. Psicologia, 20(1), 97-126.

Mikulincer, M., \& Shaver, P. R. (2007). Attachment in adulthood: Structure, dynamics, and change. New YorK: Guilford Press.

Mikulincer, M., \& Shaver, P. R. (2012).

Muraru, A. A., \& Turluic, M. N. (2012). Family-of-origin, romantic attachment, and marital adjustment: a path analysis model. Procedia - Social and Behavioral Sciences, 33, 90-94. doi: 10.1016/j.sbspro.2012.01.089

Pallini, S., \& Barcaccia, B. (2014). A meeting of the minds: John Bowlby encounters Jean Piaget. Review of General Psychology, 18(4), 287-291. doi: 10.1037/qpr0000016

Piaget, J. (1947). La représentation du monde chez l'enfant. Paris: PUF.

Piaget, J. (1948). La naissance de l'intelligence chez l'enfant.

Paris:

PUF.
Pietromonaco, P. R., \& Barret, L. F. (2000). Attachment theory as an organizing framework: A view from different levels of analysis. Review of General Psychology, 4(2), 107-110. doi: 10.1037//1089-2680.a.2.107

Scott, L. N., Whalen, D. J., Zalewski, M., Beeney, J. E., Pilkonis, P. A., Hipwell, A. E., \& Stepp, S. D. (2013). Predictors and consequences of developmental changes in adolescent girls' self-reported quality of attachment to their primary caregiver. Journal of Adolescence, 36(5), 797-806. doi: 10.1016/j.adolescence.2013.06.005

Simpson, J. A., \& Rholes, S. (2012). Adult attachment orientations, stress, and romantic relationships. Advances in Experimental Social Psychology, 45, 279-328. doi: 10.1016/B978-0-12-394286-9.00006-8

Snyder, C. R., Lopez, S. J., Shorey, H. S., Rand, K. L., \& Feldman, D. B. (2003). Hope theory, measurements, and applications to school psychology. School Psychology Quarterly, 18, 122-139.

Sroufe, A. (2005). Attachment and development: A prospective, longitudinal study from birth to adulthood. Attachment \& Human Development, 7(4), 349-367. doi:10.1080/1461673050036592

Twedt, M. (2013). A comparison of the attachment with parents and the attachment of romantic partner. Psychology Student Work, Paper13.

Vorria, P., Vairami, M., Gialaouzidis, M., Kottroni, E., Koutra, G., Markou, N., et al (2007). Romantic relationships, attachment styles, and experiences of childhood. Hellenic Journal of Psychology, 4, 281-309.

Waters, H. A., \& Waters, E. (2006). The attachment working models concept: Among other things, we build sript-like representations of secure base experiences. Attachment \& Human Development, 8(3), 185-197. doi: 10.1080/14616730600856016 